\title{
THE ROLE OF ACTIVITY OBJECTS IN CROWDSOURCED DIGITAL INNOVATION
}

\author{
Jiayuan Liu \\ University of Warwick. United Kingdom
}

\begin{abstract}
In this study, I explore how activity objects orchestrate knowledge for crowdsourced digital innovation. Adopting a mixed-methods research approach, my quantitative results from 355 questionnaires corroborate the three hypotheses, and my qualitative evidence collected from 48 interviews enriches and adds depth to my explanations. As a result, I found that by acting as a trigger for expansive learning, and a director and motivator of crowdsourcing communities, activity objects serve to facilitate the sharing, acquisition, and integration of knowledge, coordinating knowledge heterogeneity and countering its fragmentation for crowdsourced digital innovation.
\end{abstract}

\section{KEYWORDS}

Activity Objects, Crowdsourced Communities, Digital Innovation, Knowledge Orchestration

\section{INTRODUCTION}

As crowdsourcing communities have increasingly got involved in the process of digital innovation, scholars have paid more significant attention to their innovation networks (Mladenow et al., 2014). Such networks are defined as doubly distributed innovation networks, in which the organizational and technological control over product components is distributed across firms of different kinds, and where the product knowledge is distributed across heterogeneous communities and specialties (Yoo et al., 2010). However, such innovation networks also bring with them their own challenge: the knowledge heterogeneity and fragmentation (Lyytinen et al., 2015).

In order to address this challenge, a certain amount of orchestration, influence and direction is needed for the network actors to appropriately transfer, accept, and leverage knowledge without sacrificing flexibility and independence in the processes of innovation. Drawing on a network orchestration model (Dhanaraj \& Parkhe, 2006), I identify three purposeful, interrelated knowledge orchestration activities to maximize the output of digital innovation: knowledge sharing, knowledge acquisition, and knowledge integration. 
IADIS International Journal on Computer Science and Information Systems

As human activity is always mediated by cultural artefacts (Engeström, 1999), scholars suggested that activity objects could be a useful starting point in addressing the knowledge orchestration. According to Miettinen (2005), activity objects are partially emergent, partially fragmented and partially contradictory, as well as under-defined, unfolding objects in collaboration; simultaneously, they maintain the activities around the pursuit of themselves.

Although existing studies recognized the role of cultural artefacts in providing the direction, motivation and meaning for an activity (Kaptelinin \& Nardi, 2006), our understanding of how activity objects serve to orchestrate knowledge for crowdsourced digital innovation is still very limited. In order to fill this gap, I aim to explore how activity objects influence the sharing, acquisition and integration of knowledge for crowdsourced digital innovation. Thus, my research question is: how do activity objects orchestrate knowledge to coordinate its heterogeneity and counter its fragmentation in crowdsourced digital innovation?

The rest of the paper is organized as follows: in the next section, I integrate diverse bodies of literature to develop my hypotheses; then I use questionnaires to test these hypotheses, and conduct interviews to enrich the quantitative results. Last, I report my results and articulate their contributions, including theoretical and practical implications.

\section{THEORETICAL BACKGROUND}

\subsection{Conceptualizing Activity Objects in Crowdsourced Digital Innovation}

According to cultural historical activity theory, activity objects are able to mediate any human activity, by enabling purposeful action, connecting agents to their social surroundings, and embedding into the activity the history that they embody (Engeström, 1999). Simultaneously, human activity is also oriented toward a cultural artefact, recognized as a prospective outcome that motivates and directs the activity (Kaptelinin \& Nardi, 2006). Thus, a focus on activity objects can give hints as to why the crowdsourced digital innovation activity happens in the first place, and how individuals contribute their knowledge to the construction of the activity object, as well as how they attach their different expectations to this object.

Because an activity's object is recognized as emergent, fragmented, and contradictory, collective human activity is always maintained around the pursuit of a partially emergent, partially fragmented, and partially contradictory object (Nicolini et al., 2012). Hence, an activity object can be viewed as a conflict trigger and a director and motivator of the community that evolves and revolves around itself. Specifically, an activity object can act as a representation for crowdsourced digital innovation from three perspectives.

First, because an activity object is inherently multi-faceted, fragmented, and disputed, it can create a socio-material community around itself, into which "a naturally occurring and evolving collection of people" with contradictory interests, orientations and interpretations "engage in particular kinds of activity", and "develop and share ways of doing things as a result of their joint involvement in that activity" (Galagan, 1993, p. 33). As an activity object attracts heterogeneous actors with diverse knowledge boundaries to engage in the process of digital innovation, a crowdsourcing community emerges that absorbs the wisdom of each actor, and triggers reflective learning (Patil \& Lee, 2016). Because an activity object can be seen as a trigger of conflict due to the potentially contradictory nature of collective activity 
(Miettinen, 2005; Miettinen \& Virkkunen, 2005), the crowdsourcing community, that is composed of actors rooted in heterogeneous worlds with weak ties (Granovetter, 1973), is not an integrated whole in which parts move in harmony, but rather is a "community without unity", in which contradictions and expansive learning abound at the same time (Nicolini et al., 2012).

Second, an activity object is partly predetermined and partly emergent, reflecting the originally embedded, and constantly evolving, interests of the actors involved (Nicolini et al., 2012). As a cultural artefact serves as an object of a crowdsourced digital innovation activity, it enables the collective action to emerge around it according to a shared goal; simultaneously, it is also the result of the practices and expectations of the crowdsourced communities that gather around it (Miettinen, 2005). With widely distributed, heterogeneous actors engaging in the process of digital innovation, an activity object is able to help them "find the signal in the noise" while avoiding irrelevant content (Paul et al., 2012). Thus, such an object acts as a moving target with the capacity to direct the collective activity (Miettinen, 2005).

Third, as Leont'ev (1978, p. 66) emphasized, "the object of an activity is its true motive". An activity object is able to motivate its crowdsourcing communities to continually engage in the process of digital innovation, fuelling the collective activity. Based on the social exchange theory, which suggests that individuals take actions according to their calculated benefits and costs (Lanham, 2006), the motives for actors devoting themselves to crowdsourced activities can either be extrinsic or intrinsic (Choudhury et al., 2014).

Actors who contribute high-quality knowledge to crowdsourced digital innovation, expect to improve their reputation as a form of extrinsic reward (Jin et al., 2015). Besides reputation, attention, which has become a scarce resource in the information age, is another significant extrinsic motivator (Lanham, 2006). Drawing on the idea that, in the 'attention economy', information consumes its recipients' attention, Lanham (2006) described how social communities seek to compete for each other's attention. In this way, network actors are extrinsically motivated to exchange their knowledge for reputation and attention, which can be explained in terms of the concept of desire, drive or struggle for recognition (Hegel, 1977).

Specifically, social recognition is perceived as a primary source of personal identity, which is especially significant in crowdsourced activities where division of labour is a source of individuality (Miettinen, 2005). As social recognition is identified as "esteem achieved in community life", any recognition of individuals' uniqueness is positively related to the future contribution they will make to the collective activity (Miettinen, 2005, p. 62). This is also true in highly distributed, virtual, crowdsourcing communities, where the recognition, acknowledgement and reward for the contributions that members make is important in assigning identity to themselves and maintaining their communities (Lerner \& Tirole, 2001).

This kind of social recognition can be achieved by objectified actions and objects (Kojève, 1969). That is, actors pursue recognition for their actions and these actions' objectifications both within a cultural activity and in wider communities (Miettinen, 2005). This is because, as actors become increasingly recognized by participating in a collective activity, such participation can be objectified in the products of their actions, with their achievements constituting the objectified demonstration of their capability to contribute to their communities and the target activity (Knorr-Cetina, 1997). Therefore, activity objects are able to realize and demonstrate the unique contributions that members make, which continuously fuels their participation in and contribution to both the activity and their communities (Miettinen, 2005). 
IADIS International Journal on Computer Science and Information Systems

With extrinsic benefits providing the main motivations for crowdsourcing communities to initiate the behaviour of knowledge contribution for innovation, intrinsic rewards involved in social exchanges that emphasize unspecified obligations, such as social affiliation and feelings of belonging, trust and self-actualization, carry more weight in their motivation of continuous engagement in the community (Sigala \& Chalkiti, 2015). Nicolini et al. (2012) pointed out that activity objects can trigger intimate emotional attachment that is not restricted to individuals but is performed as an engine of solidarity, a collective obligation and an emotional affiliation, constituting a morally binding force among community members. In this way, the object of an activity can provide a "family of invisible friends" with a "home" in which a sense of loyalty can be engendered in committing to the digital innovation goal (Abrams et al., 2003). Such community affiliation, triggered by the activity object, intrinsically motivates crowdsourcing communities to identify themselves with the communal goal, while putting their self-interests aside, which fuels the impetus for them to commit to the totality.

\subsection{Knowledge Orchestration for Crowdsourced Digital Innovation}

As digital innovation is seen as inherently layered, it increasingly pushes heterogeneous actors to connect across multiple boundaries to create new value-in-use, forming a crowdsourced innovation network (Huang et al., 2017). However, such a network can lead to the knowledge becoming too fragmented and heterogeneous. Facing this problem, Dhanaraj \& Parkhe (2006) suggested that a certain amount of coordination, influence and direction is needed for crowdsourcing communities, to transfer, accept and leverage knowledge without sacrificing flexibility in the processes of innovation. Specifically, I define knowledge orchestration as the combination of knowledge sharing, knowledge acquisition and knowledge integration.

First, knowledge sharing concerns the ease with which knowledge is transferred within a network (Dhanaraj \& Parkhe, 2006). Knowledge transfer is predominantly referred to in the network literature as an 'asset' that carries value for a network (Nahapiet \& Ghoshal, 1998). Particular emphasis is placed on standardizing or establishing compatible methods of communication to facilitate the sharing of this form of intellectual capital across the 'syntactic' boundaries (Carlile, 2002), from one actor to the next.

Second, when the transferred knowledge is complex and there is not clarity of purpose, then the challenge shifts to the acquisition of the knowledge, where a 'semantic' approach (Carlile, 2002) is needed to recognize the different ways in which each actor interprets and accepts the disseminated message.

Finally, knowledge integration occurs - and the full potential of the innovation network is only realized - if and when the heterogeneous knowledge resources are combined together and transformed into an innovation (Crossan \& Inkpen, 1995). Carlile (2002) proposed a 'pragmatic' view of knowledge, and in crowdsourced digital innovation, this poses a challenge for the network actors, namely, fully exploring their unique local context, without losing their ability to interrelate and transform different types of 'hard-won' knowledge into an innovation that transcends its customary pragmatic boundaries. 


\subsection{Hypothesis Development}

Drawing on the literature, I develop three hypotheses to explore how activity objects orchestrate knowledge for crowdsourced digital innovation. Specifically, I examine the impact of activity objects on knowledge sharing, knowledge acquisition and knowledge integration. The development of these hypotheses can be summarized as follows.

According to Nicolini et al. (2012), activity objects can create a crowdsourcing community, that is not an integrated whole where parts move in harmony, but is rather a community without unity, in which conflicts and contradictions abound. Because a wide collection of conflicting interpretations and contradictory assumptions regarding problems enables community members to search for optimal solutions, evaluate diverse methods, and debate and filter out invalid answers, their group-thinking is decreased and a reflective learning takes place (Scarbrough et al., 2004). Such expansive learning, which involves overcoming heterogeneous boundaries in the transfer and flow of knowledge arising from pre-established cognitive divisions of the community actors involved (Scarbrough et al., 2004), deepens their communication intensity and promotes their information diffusion, thereby increasing their opportunity to share and mobilize widely dispersed pieces of knowledge for crowdsourced innovation (Sigalaa \& Chalkiti, 2015).

In addition, Boland et al. (2007) highlighted the significance of expansive learning in the transfer of knowledge by proposing the concept of a "trading zone", that is, a cognitive and physical area in which actors with individual innovation trajectories can innovate. Specifically, when crowdsourcing community boundaries overlap or cross during the process of mutually communicating, discussing, negotiating, and innovating (Boland \& Tenkasi, 1995), a trading zone may emerge in which a high level of learning flows in multiple directions, and knowledge can travel from one community into another freely, facilitating the mobilization and sharing of knowledge (Boland et al., 2007).

Furthermore, as a socially interactive cultural artefact, an activity object can drive socio-emotional forces such as trust, commitment and loyalty, and trigger an ethical community culture, conducive to information diffusion and knowledge mobilization (Tsai, 2001). Specifically, Abrams et al. (2003) emphasized the role of trust in alleviating the fear of risk and creating an atmosphere in which the sources and recipients are less inclined to engage in cost-benefit calculus, and more willing to credit each other's viewpoints and to exchange information with others. Such trust is also significant for the transfer of tacit knowledge that is hard to communicate as readily as information, and this is particularly true in the context where crowdsourcing communities are virtual and widely distributed with consequent difficulties in enforcing, measuring or monitoring their implicit knowledge contributions (Davenport \& Perusak, 1998). Hence, I hypothesize that:

\section{H1: Activity objects foster knowledge sharing for crowdsourced digital innovation.}

As an activity object creates a community around itself with the passage of time, knowledge acquisition is facilitated, because the best way to access knowledge is to interact with the community (Mandl et al., 1996). From the perspective of communities of practices, knowledge assimilation is not about absorbing information, but rather is about becoming a part of a community, which is a social process built around informed participation (Engeström, 1991). In crowdsourcing communities where learning is identified to be nothing more than accepting socially shared beliefs and practices, activity objects foster the social 
IADIS International Journal on Computer Science and Information Systems

process of enculturation, promoting the acquisition of knowledge that includes not only procedural and declarative expertise but also social beliefs and values (Lave \& Wenger, 1991).

In addition, because activity objects act as triggers of negotiation and conflict (Miettinen \& Virkkunen, 2005), they serve to spur expansive learning, a powerful driver for the acquisition and assimilation of knowledge. From the perspective of boundaries of different communities of practice, one platform by which expansive learning occurs is through addressing these boundaries of different communities (Lave, 1992). When members of different communities learn from one another, and have to incorporate distributed pieces of knowledge from each member for problem-solving, this process involves the change of their identity, through which expansive learning as well as knowledge acquisition can occur (Merry, 1995).

Meantime, friction is likely to happen between heterogeneous actors at the boundaries of different communities of practices, as members as a whole iteratively affect each other by building and modifying the changes in each other's identities (Stamps, 1997). Such inter-community boundaries are the places where knowledge creation and acquisition occur, where diverse actors engage in an expansive learning to compare and contrast their viewpoints with each community, thereby fostering the assimilation of 'knowledge-in-context' in terms of their various requirements (Paul et al., 2012). Hence, by revealing cognitive conflicts and triggering expansive learning among diverse communities, activity objects serve to foster knowledge elicitation and promote intellectual exploration, thereby enhancing their opportunity to generate high-quality knowledge (Rennstam, 2012).

Furthermore, activity objects can attach the social desire for recognition and approval to themselves (Miettinen, 2005), which is perceived as a primary source of motives for the members to make contributions to their communities. As the number of actors motivated to contribute their knowledge to crowdsourced digital innovation increases, activity objects serve to broaden the knowledge that improves the conditions essential for the acquisition of knowledge for innovation (Davenport \& Perusak, 1998). Specifically, with a community of practice that revolves and evolves around an activity object, this object acts as a trigger to attract broader communities and thus develop wider (weak) ties (Granovetter, 1973). Such ties, critical for the transmission of novel information, expose the community members to a diversity of external contacts that increases the breadth and depth of their knowledge base, and provides extra opportunities to acquire knowledge (McEvily \& Zaheer, 1999). Hence, I hypothesize that:

H2: Activity objects foster knowledge acquisition for crowdsourced digital innovation.

Drawing on the potentially contradictory nature of collective activity (Miettinen \& Virkkunen, 2005), activity objects trigger contradictions and conflicts that can spur expansive learning (Engeström, 1987), essential for the integration of knowledge. Specifically, the cognitive conflicts induced by the object of an activity enable the community members to realize their incomplete ideas, appreciate dissenting views, resolve issues of controversy and create optimal solutions, resulting in a high level of expansive learning (Sockalingam, 2000). Such learning plays a critical role in overcoming pragmatic boundaries to the transfer of knowledge resulting from the divisions in practice associated with differing in political interests (Carlile, 2002). In this way, by revealing contradictions and clarifying interdependencies among crowdsourcing communities (Garrety \& Badham, 2000), activity objects engage these communities in a process of expansive learning, leading to the integration of heterogeneous knowledge at the interface of community boundaries for crowdsourced innovation (Boland et al., 2007). 
Furthermore, activity objects can provide the members who pursue reputation improvement with extrinsic motivators for continually contributing to the community. By inducing the desire for recognition among the actors, the object of an activity creates an affective relationship with its actors, with which they are engaging in a collective activity (Miettinen, 2005). As more members are motivated to participate in high-order reflective learning, activity artefacts facilitate the exchange of information and the mobilization of cognitive resources that foster the assembly, combination and recombination of heterogeneous pieces of knowledge for crowdsourced innovation.

The additional extrinsic motivator that an activity object can provide is attracting attention, by effectively attaching its community members' goals, motives and expectations to itself to recognize, acknowledge, and reward user contributions (Jin et al., 2015; Miettinen, 2005). By providing such an extrinsic motivator, activity objects serve to get more actors access to a variety of knowledge and thus increase their opportunity to leverage the existing knowledge for the creation of novel associations (McEvily \& Zaheer, 1999). Hence, I hypothesize that:

H3: Activity objects foster knowledge integration for crowdsourced digital innovation.

\section{RESEARCH METHODS AND RESULTS}

\subsection{Zhihu: A Representative Activity Object}

In this study, I selected "Zhihu"- a Chinese question-and-answer (Q\&A) website, where widely distributed actors across heterogeneous communities create questions, crowd-source the search, and vote for high-quality answers- as a representative object of crowdsourced digital innovation activity for three reasons.

First, as a community platform, Zhihu is able to attract a number of actors with heterogeneous expertise and potentially contradictory interests to engage in the target activity of asking and answering questions, thus creating a crowdsourcing community around it for digital innovation. Because, as a social network website, Zhihu is inherently disputatious, it can also act as a trigger of conflict for its crowdsourcing communities, a place where they can engage in an expansive learning by posting challenging Q\&As, searching for topics of interest, voting answers up or down and commenting on controversial content.

Second, as highly distributed, heterogeneous online actors engage in the process of asking and answering questions, Zhihu is able to help them "find the signal in the noise", while avoiding irrelevant content (Paul et al., 2012). Specifically, Zhihu supports a voting service such that more authoritative answers can get up-voted to the top of the answer list, while less helpful answers can get down-voted and eventually filtered out (Patil \& Lee, 2016). In this way, Zhihu directs the process of separating high-quality answers from ill-formed alternatives on the basis of the numbers of votes received. Because answers produced by primary sources of information with first-hand testimony or direct evidence pertaining to a question are generally perceived as authoritative (Harper et al., 2008), in voting for high-quality content with primary source knowledge, crowdsourcing communities often judge others' reputations according to their online profiles. This information, which may include background expertise, past contribution history, and online popularity, provides a context for Zhihu users to verify an answer's validity, thereby helping them vote up valuable answers (Paul et al., 2012). The 'invite' function of Zhihu also allows users to tag specific individuals in relation to particular 
IADIS International Journal on Computer Science and Information Systems

questions to try and ensure that these questions are answered by the most authoritative experts. Hence, Zhihu directs the crowdsourced digital innovation activity by leveraging appropriate social connections to help users acquire high-quality answers.

Third, Zhihu serves to motivate its crowdsourcing communities to continually contribute to the collective activity of asking and answering questions, by providing them with extrinsic and intrinsic incentives. In terms of extrinsic incentives, because online users are motivated to exchange their high-quality knowledge for reputation and attention, Zhihu is able to recognize, acknowledge and reward the user contributions via its voting mechanism (Jin et al., 2015). Because knowledge contributors desire being deemed valuable to the community, an up-vote provides gratification for their customized answers, which fuels their continued participation in the community. In terms of intrinsic incentives, Zhihu provides a place where feelings of belonging, loyalty and trust can be engendered among a 'family' of friends. Such community affiliation intrinsically motivates the community to commit to the digital innovation activity.

\subsection{Quantitative Research}

In this study, I used web-based surveys to test the hypotheses. Specifically, I conducted the survey via the SurveyMonkey, and measured the items using seven-point Likert scales ranging from 1 (strongly disagree) to 7 (strongly agree). The study population consisted of 500 Zhihu users in the groups of digital product innovation, software design and apps use. I distributed 500 questionnaires and deemed 355 usable for the quantitative analysis. To collect reliable data, I asked key informants to respond to the surveys, typically well-known Zhihu users with a good reputation and large numbers of up-votes, who frequently use their expertise to provide high-quality answers and leverage their social connections to obtain useful knowledge. Of the response population of 355, 301 had been registered on Zhihu for more than three years; those with a PhD numbered 105 (29.6\%), while those with industrial experience in digital innovation accounted for $65 \%$. The measures are developed as follows.

Zhihu as an activity object (ZH). Drawing on the literature, an activity object is able to perform as a trigger of conflict, a director and motivator of crowdsourced communities (Miettinen, 2005; Miettinen \& Virkkunen, 2005; Nicolini et al., 2012). In this study, I regarded Zhihu as a representative activity object and measured ZH using four items. Specifically, I asked those Zhihu users to what extent Zhihu has (1) developed a community around the digital innovation activity, in which they crowdsourced to engage in expansive learning; (2) directed them to select the best answer while filtering out unpopular ones; (3) recognized and rewarded their contributions to encourage them to ask and answer questions; (4) induced feelings of trust and belonging to motivate them to commit to their community and digital innovation activity.

Knowledge sharing (KS). According to Dhanaraj \& Parkhe (2006), knowledge sharing concerns the ease with which knowledge is transferred and deployed within a network. Specifically, I used two items for measuring the ease of knowledge transfer from a source to a recipient. The two items were illustrated as "It is easy for me to explain to the other users a key idea, concept, or theory as well as new developments in my area of expertise", and "It is easy for me to follow and disseminate the other users' ideas either orally or via information technologies". In addition, I referred to Hall et al.'s (1975) Levels of Use scale to measure the ease of knowledge deployment within a network for creating innovations. Specifically, I used two items to measure to what extent respondents pay attention to day-to-day use of their expertise for addressing applied problems to spur innovations, and to what extent respondents 
stabilize the application of their expertise to address applied problems for creating innovations.

Knowledge acquisition (KA). I adopted the measurements of the construct of absorptive capacity to measure the ease with which knowledge is accepted, acquired and exploited by the Zhihu users, by referring to Seo et al.'s (2015) 4-item-scale designed for measuring individual absorptive capacity and Gluch et al.'s (2009) 10-item-scale designed for measuring the routines for acquiring and assimilating knowledge. Specifically, I used three items to measure to what extent respondents recognize and accommodate the different ways in which each actor interprets and accepts the disseminated message, and to measure the degree to which respondents receive and assimilate the shared information and knowledge resources, increasing their existing knowledge base.

Knowledge integration (KI). According to Schutz et al. (2009), knowledge integration concerns the extent to which the network members leverage their diverse domains of expertise for creating innovations. Specifically, I used two items for measuring the KC construct: "I have established a full understanding of each user's expertise as well as how each user's knowledge should be coordinated", "I have carefully interrelated practice-based expertise and actions to each other for strategic responses to changes in the external environment". Furthermore, I draw from the knowledge integration instrument developed by Ou et al. (2008) for measuring KC. More specifically, I adopted two items to measure to what extent respondents understand how two or more ideas, concepts or theories across different specialties interact in an innovation context, and to measure the degree to which respondents recognize potential connections between ideas, concepts or theories across different specialties, and elaborate the nature of these connections specific to an innovation context.

\subsection{Qualitative Research}

In this part of the research, I used interviews to explore how Zhihu affects the sharing, acquisition and integration of knowledge, enriching the quantitative results with supplementary evidence. Specifically, I conducted 48 semi-structured interviews, each lasting for 45 minutes. To collect the most reliable data available, I selected those Zhihu users with a high number of up-votes for their answers and a good reputation in their communities, suggesting that they can seek and provide high-quality answers, and are able to recognize the elements affecting the process of knowledge-orientated innovation. In terms of their demographic contexts, 22 of these users focused on developing digital games, and 26 focused on designing mobile apps. Of these 48 respondents, 12 held a $\mathrm{PhD}$, and 29 had industrial experience in digital innovation.

Moving to data analysis, I coded the interview transcripts to identify key themes and categories pertaining to each of the questions. By way of example, for one question, I analyzed the full transcripts, attempting to find connections between the answers and the motivations. After coding different transcripts, I identified several categories of answers and the corresponding motivations given by various respondents. Then I went back to the transcripts to ascertain how many interviewees' viewpoints belonged to each category. In addition, I also used some initial codes based on the known theoretical concepts in the literature to categorize the transcripts. During the process of recursively moving back and forth between original recordings and transcripts, my appreciation of the link between Zhihu, knowledge sharing, knowledge acquisition and knowledge integration emerged progressively. In moving between data and theories, I used N-Vivo to check whether the emerging themes were supported by the data and whether theories helped make sense of the empirical evidence. 
IADIS International Journal on Computer Science and Information Systems

\subsection{Quantitative Results}

In terms of a measurement model (Table 1), I first conducted an exploratory factor analysis of the four measures $(\mathrm{ZH}, \mathrm{KS}, \mathrm{KA}, \mathrm{KI})$, by using a principal axis factoring analysis with Oblimin oblique rotation with Kaiser normalization rotation. Specifically, KMO was 0.786, indicating that the data was suitable for factor analysis. In addition, the data showed support for the four factors, which had eigenvalues greater than 1 and explained $96.129 \%$ of the variance. Furthermore, the measures suitably represented the four factors whereby all the primary loadings exceeded 0.709. Finally, the Cronbach's alpha was 0.906 , implying a high reliability of internal consistency.

I also conducted a confirmatory factor analysis to estimate the model using SPSS Amos, consistent with Anderson and Gerbing's (1988) two-step approach. First, all indexes displayed a good fit with the model: the observed CMIN was 421.15 with 399 DF, the NFI was 0.990, CFI was 0.980, and RMSEA was 0.021, suggesting a good model fit. Second, I examined the convergent validity by testing the significance of the factor loadings and their gap to the S.E. (Koufteros, 1999). All item loadings were above the suggested cut-off of 0.6 (Hair et al., 1998), with a strong significance level. Additionally, all the S.E. values were around 0.1, indicating that all the items had a clear relationship with their own latent variables. Furthermore, all the CR values of the latent variables were above the criterion of 0.7 (Hair et al., 1998), showing a good convergent validity. Finally, all the square roots of the AVE shown on the diagonal of the correlation matrix were greater than the off-diagonal construct correlations, implying a good discriminant validity (Koufteros, 1999).

With regard to the structural model (Figure 1), I used Amos to test the hypotheses. All the paths were significant, supporting the three hypothesized relationships. The coefficients of Zhihu were positive and significant for knowledge sharing $(\beta=.23, \mathrm{p}<.01)$, knowledge acquisition $(\beta=.53, p<.001)$, and knowledge integration $(\beta=.19, \mathrm{p}<.01)$. This support H1, H2 and $\mathrm{H} 3$, indicating that, as an activity object, Zhihu fosters the sharing, acquisition, and integration of knowledge for crowdsourced digital innovation.

Table 1. Measurement model

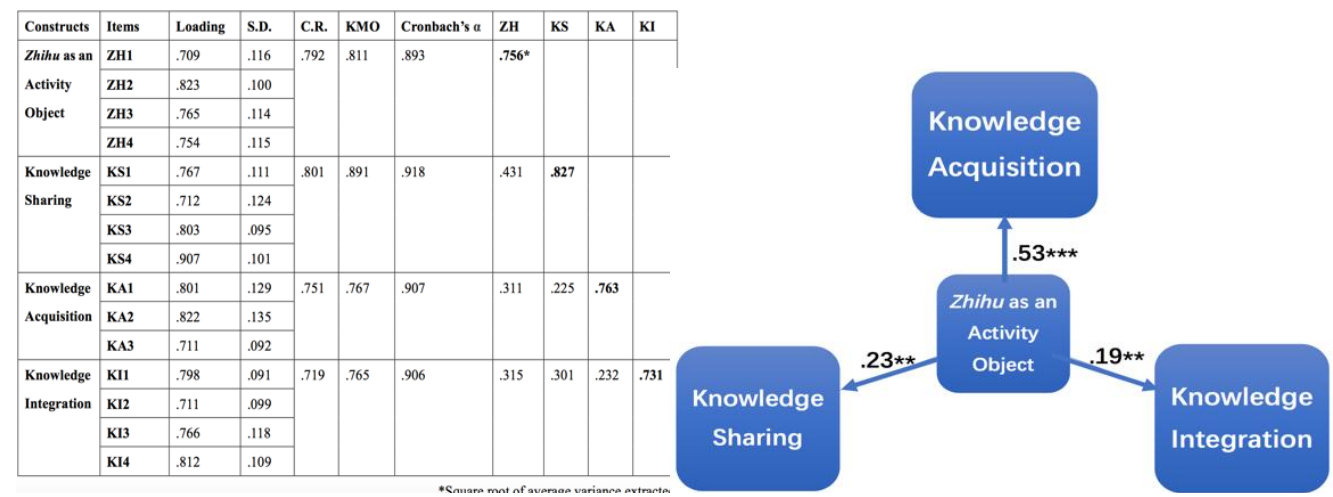

Figure 1. Structural model 


\subsection{Qualitative Results}

The quantitative result for hypothesis H1 was corroborated by my interview data. Specifically, I found that Zhihu created a crowdsourcing community, whose trajectory was shaped by different knowledge relationships, and where contradictions abounded to trigger expansive learning. As one user said: "One question can bring us together. We see the question first, then we use our expertise to answer the question. We also look at others' answers, compare and contrast their answers with ourselves. It is normal that we have different views and we answer the question from different perspectives. It is these different interpretations that make us evaluate multiple methods, seek optimal solutions, and abandon invalid answers."

As high degrees of expansive learning overcame heterogeneous barriers to the transfer of knowledge arising from pre-established divisions of crowdsourcing communities, knowledge travelled more freely from one community into another, promoting the mobilization of dispersed pieces of knowledge for innovation. This was reflected in an interview: "I follow a topic called creative app design, where there are many Q\&As concerning the feasibility of novel apps based on iOS 10, like their potentials and problems. There are over 200 persons under this topic, who share an interest in digital innovation based on iOS 10. We often engage in a dialogue to discuss, argue and debate with each other regarding one specific question, and this behaviour can deepen our communication. Although we have different expertise, we can quickly develop a shared understanding of optimal problem-solving."

In addition, some interviewees believed that Zhihu triggered socio-emotional forces among them, which promoted the information flow and fostered the knowledge diffusion. Specifically, one user highlighted how Zhihu created an ethical and cohesive culture within his community, in which he was willing to answer questions, credit the others' ideas and openly share valuable answers: "Because we are all interested in iOS 10.1.1, it is easy for us to discuss the function of this system, its potential and its bugs. So, it is not weird that during the process of asking and answering questions together, we can generate a high trust in each other's willingness and competence to solve problems."

My qualitative data similarly made further sense of hypothesis H2. Most interviewees highlighted the role of Zhihu in separating valuable answers from alternatives in directing them in the acquisition of high-quality knowledge. Specifically, Zhihu provides a space where valuable answers generally get up-voted to the top of the answer list, while irrelevant content gets down-voted and eventually filtered out.

The 'invite' mechanism of Zhihu also leveraged proper social connections to help its users gain authoritative answers from primary information sources, by allowing them to tag persons who have direct evidence on specific questions. As one interviewee highlighted: "Zhihu is an amazing place where you can approach a diversity of experts who handle the most primary information sources in different domains. For example, when you ask a question about comparing Huawei Mate 9 with iPhone 7, it is possible that it will be a designer working within Huawei who provides you with a (very authoritative) answer."

Nevertheless, some interviewees argued that an answer from such primary knowledge sources does not necessarily represent a higher quality response. In order to find the "real" signal in the noise, Zhihu engages its users in a reflective dialogue, where they are empowered to access a pool of diverse answers, compare and contrast their answers with others, and negotiate their perspectives mutually, fostering the assimilation of 'knowledge-in-context' in terms of their various requirements (Paul et al., 2012). As one user reported: "Many people up-vote the answers from primary information sources, but I am not one of them. I only 
IADIS International Journal on Computer Science and Information Systems

respect logic. If the logic of an answer convinces me, I will give it an up-vote. If not, I tend to argue with the answer provider, in order to create a better answer. Vice versa, I like to discuss with those who question my answers, and to conduct some self-reflection."

In addition, some interviewees believed that Zhihu propelled them to continually engage in the process of creating questions and formulating answers, by providing them with reputation. Specifically, I found that Zhihu's function of assessing the helpfulness of responses attracted answers from many users pursuing reputation improvement. This was reflected in one interview: "When I receive up-votes from others, I can really feel their approval of my answer. This gives me more confidence to answer other questions, in order to achieve more up-votes. I want to use my expertise to answer questions and help others solve problems, but I also want social recognition for my contribution. These two requirements don't conflict with each other; on the contradictory, each takes what the other needs." As increasing knowledge is contributed via the answer posting, Zhihu broadens the knowledge available to its users, fostering conditions essential for the acquisition of knowledge for innovation.

The quantitative results for hypothesis $\mathrm{H} 3$ were also enriched by my interviews. Most users highlighted that Zhihu brought them into contact with cognitive conflicts that encouraged them to realize their incomplete ideas, embrace dissenting views, resolve issues of controversy, and seek optimal solutions. Thus, one interviewee reported: "Although we share a common interest, we have different experiences, we work in different domains. It is normal that we have various ways of thinking, framing problems and solving problems. It is also normal that we perceive and answer questions differently". And another stated: "Conflict is not necessarily a bad thing. It can make me realize what my problem is, and where my idea has gone wrong, so that I have a chance to correct. For the whole community, it can engage us in a reflective, open dialogue, which is also a positive thing."

Hence, these interviewees were empowered to engage in a significant learning critical to overcoming pragmatic barriers to the transfer of knowledge resulting from the divisions in practice, and conducive to the transformation of knowledge for innovation. This was seen in one interview: "When we group together around one specific question that we all are interested in, we can have a full picture of each other's expertise, thereby recognizing the knowledge needed for answering this question, tinkering with various pieces of knowledge in parallel, and developing a shared understanding for problem-solving. "

Furthermore, Zhihu provides its users with attention as an extrinsic incentive, by using its voting function to recognize and reward user contributions. To compete for each other's attention, Zhihu users tended to post more challenging questions to attract wider communities to answer them, and to build more ties that help the users to access a wider variety of knowledge and leverage more existing knowledge for innovation. As one interviewee said: "Sometimes, I deliberately organize my question in an unexpected way and tag those famous 'big shots', in order to attract their attention and increase my popularity. Vice versa, I always answer selectively and intelligently, attempting to achieve more up-votes and attention."

\section{DISCUSSION AND CONCLUSIONS}

My quantitative and qualitative results demonstrate how activity objects orchestrate knowledge, and thus I make two contributions. My first contribution is to recognize Zhihu as a trigger for expansive learning, and a director and motivator of crowdsourcing communities in 
facilitating the sharing, acquisition and integration of knowledge, thereby presenting a novel private-collective model for crowdsourced digital innovation with an integration of personal investment and collective action (Trompette et al., 2008).

Specifically, this private-collective model for crowdsourced digital innovation involves an effective coordination between mutually dependent collective action and personal investment (Trompette et al., 2008). With regard to collective action, I identified the ability of Zhihu to direct the qualifying process to ensure the attainment of high-quality answers, and even support the shift from highly credible ideas to potential innovation opportunities. This co-evaluation process combines quantitative and qualitative means.

For the quantitative measures, the crowd can evaluate an answer's usefulness via voting, with more authoritative answers getting up-voted, and less popular ones getting down-voted and filtered out. By attributing a "like" to an answer to indicate how many users favour it, Zhihu directs the process of separating high-quality content from alternatives, which saves significant time and allows crowdsourcing communities to make more accurate decisions.

For the qualitative means, the crowd may offer their various opinions on certain questions, comment on answers given or convert novel ideas into feasible plans. For example, Zhihu's 'invite' mechanism enables the crowd to tag users in relation to certain questions to obtain more useful answers. Thus, I highlighted the capacity of Zhihu to shape the collective activity and "find the signal in the noise" for crowdsourced digital innovation (Paul et al., 2012).

In terms of personal investment, I recognized the ability of Zhihu to encourage the investment of the diverse knowledge resources of intrinsically and extrinsically motivated users in the creation of Q\&As, promoting the aggregation of added-value contributions to crowdsourced digital innovation (Trompette et al., 2008).

For the extrinsic incentives, I emphasized Zhihu's ability to provide its users with a space to compete for each other's attention and promote their future engagement, by recognizing and rewarding their differing contributions, based on the fact that online communities tend to exchange their knowledge for attention. As Lanham (2006) indicated, social recognition indeed dominates free-riding incentives, and a member with a larger audience size may contribute more to the community.

I also highlighted the capacity of Zhihu to give its users intrinsic incentives. Whereas crowdsourcing communities are seen as densely interconnected networks of actors, Zhihu not only offers a space for knowledge orchestration, but it also encourages reciprocal behaviours by identifying, detailing and highlighting user contributions. Thus, Zhihu creates a sense of community, builds a tone of collaboration, and concentrates shared norms of trust, gratitude and respect that members have toward each other to motivate them to contribute without an a priori specified reward in sight. To illustrate, Zhihu's security policy helps its users, who disclose their personal information online, build trust that improves their perceptions of the congruent values within their communities.

For my second contribution, the focus on the independent role of an activity object as a trigger for expansive learning and a director and motivator of knowledge orchestration contributes a novel understanding of the roles of material artefacts and humans in crowdsourced digital innovation. In contexts where digital technology has democratized the communication tools, where product and industry boundaries have become blurred, and where decentralized crowdsourcing communities have emerged to leverage mutual intelligence for innovation, the danger lies in knowledge being too fragmented and heterogeneous (von Hippel, 2005). 
IADIS International Journal on Computer Science and Information Systems

Orlikowski (2007) perceived material artefacts as technical objects or managerial instruments, which are generally utilized to sustain and support the daily work in the hands of managers who speak on their behalf. Vázquez (2006) emphasized the active role of managers as the agents of control through supervision or normative means. Drawing on this work, I have highlighted the active role of these material artefacts in enabling networks of actors to freely share, acquire and integrate knowledge, and in mediating dialogue between differing perspectives, maximizing the wisdom of the crowd for digital innovation. In this way, my research provides a new insight into how material artefacts can coexist with other types of formal managerial control in a beneficial manner, geared toward coordinating the fragmented and heterogeneous knowledge for crowdsourced digital innovation.

Specifically, I gave prominence to activity objects that have been applied to collaborative development within virtual communities of practice (Hemetsberger \& Reinhardt, 2009), and linked crowdsourcing communities to digital innovation. 'Crowdsourcing', combining 'crowd' in terms of 'the wisdom of crowds' and '(out)sourcing' in the sense of opening up the R\&D process to a distributed network of heterogeneous actors via an open call, is a key trend that has been studied by many scholars (Bayus, 2013; Howe, 2006). Communities, seen as the basic organic force, are essential for achieving network effects (Surowiecki, 2004). By viewing crowdsourced digital innovation as an object-oriented, culturally mediated and collective human activity (Engeström, 1999), I presented the ability of activity objects to orchestrate knowledge by providing expansive learning, direction, and motivation for the crowdsourced digital innovation activity.

More specifically, I demonstrated how an activity object creates a crowdsourcing community around it, and how contradictions abound to trigger expansive learning, conducive to the absorption of collective wisdom for digital innovation (Nicolini et al., 2012).

In addition, I highlighted an activity object's ability to motivate the members of its community, by recognizing their desire for social recognition and a struggle for personal identity (Hegel, 1977), by attaching "esteem achieved in community life" (Miettinen, 2005, p. 62) to it and by objectifying community members' participation in the products of their actions, whereby their achievement constitutes the objectified demonstration of their capability to contribute to their community and the target activity (Miettinen, 2005). Thus, activity objects are able to recognize, acknowledge and reward the contributions of community members, continuously fuelling their participation in and contribution to the activity and their communities. This is very true in highly distributed, virtual crowdsourcing activities, where division of labour is a source of individuality (Miettinen, 2005).

Besides social recognition, I also emphasized the capacity of an activity object to trigger emotional attachment and intrinsic obligations- such as feelings of belonging and trust- that are not restricted to individuals but operate as an engine of solidarity among the members of its community (Nicolini et al., 2012). Hence, the activity object provides a "family of invisible friends" with a "home", where they are committed to crowdsourced digital innovation.

In terms of theoretical implications, by treating Zhihu as an activity object that creates crowdsourcing communities around it for digital innovation, I add to the work of Mladenow et al. (2014) and Trompette et al. (2008), who linked crowdsourcing to open innovation and recognized a community platform as an "interesting" subject for scholars. My focus on Zhihu's role as a director and motivator presents a novel private-collective model for crowdsourced digital innovation, with the integration of collective action in distinguishing high-quality content from alternatives, and the personal investment of fragmented and heterogeneous knowledge resources. From an interdependency perspective, this activity object 
and its crowdsourcing community waver between centralization and distribution of power in the control of the collective activity (Trompette et al., 2008). Thus, my study has a theoretical implication for the development of new collaboration rules among virtual crowdsourcing communities, for managing tensions to trigger expansive learning, for identifying extrinsic and intrinsic incentives to enhance individual involvement, and for establishing the collective brain to direct the innovation activity.

I also furnish two practical suggestions. First, my focus on Zhihu's director role has a practical implication for the design of such a Q\&A website. By emphasizing Zhihu's capacity to select the best answers, generally from primary information sources, I suggest that such community platforms should promote the creation of social networks based on a real-name registration policy, which provides users with improved credibility when judging others' authoritativeness (Paul et al., 2012). I also found that users tend to judge others' reputation according to their past actions and contributions; thus, I suggest such Q\&A sites to make users' online histories easier to discover, which is especially significant for those websites without complex algorithmic mechanisms for signalling user reputation (Paul et al., 2012).

Second, for those firms aiming to cooperate with crowdsourcing communities to leverage differentiated cognitive resources into something that creates novel meaning, my study provides a practical implication for the identification of motivation to encourage involved actors to contribute to crowdsourced activities. Specifically, as online users tend to exchange their knowledge for attention, I suggest those senior managers to promote such social websites as a marketplace, which connects users' needs for attention and knowledge, thereby motivating their involvement. For example, it is a good idea to leverage the response mechanisms of such social platforms to recognize user contributions (Wasko \& Faraj, 2005). I also suggest those leaders to develop a network macro-culture, which could be seen as a governance mechanism, to align the efforts of crowdsourcing users and support the safeguards against potential actor malfeasance.

\section{REFERENCES}

Abrams, L. C., Cross, R., Lesser, E. and Levin, D. (2003), "Nurturing Interpersonal Trust in Knowledge- Sharing Networks," The Academy of Management Executive, 17 (4), 64-77.

Anderson, J. C. and Gerbing, D. W. (1988), "Structural Equation Modeling in Practice: A Review and Recommended Two-Step Approach,” Psychological Bulletin, 103 (3), 441-423.

Benkler, Y. (2006), The Wealth of Networks: How Social Production Transforms Markets and Freedom, New Haven, CT: Yale University Press.

Boland, R. J., Lyytinen, K. and Yoo, Y. (2007), "Wakes of Innovation in Project Networks," Organization Science, 18 (4), 631-647.

Carlile, P. R. (2002), “A pragmatic view of knowledge and boundaries: Boundary objects in new product development," Organization Science, 13 (4), 442-455.

Choudhury, S., Alani, H. and KMI, U. (2014), "Exploring user behavior and needs in Q\&A communities," in Proceedings of the European Conference on Social Media, Brighton, 80-89.

Davenport, T. H. and Perusak, L. (1998), Working knowledge, Boston, MA: Harvard Business School.

Dhanaraj, C. and Parkhe, A. (2006), "Orchestrating innovation networks," Academy of Management Review, 31 (3), 659-669.

Engeström, Y. (1987), Learning by Expanding: An Activity-Theoretical Approach to Developmental Research, Orienta Konsultit, Helsinki, Finland. 
IADIS International Journal on Computer Science and Information Systems

Engeström, Y. (1999), “Activity theory and individual social transformation,” in Perspectives on activity theory, $\mathrm{Y}$.

Galagan, P. A. (1993), "The search for the poetry of work," Training and Development 47 (10), 33-37.

Gold, A. H., Malhotra, A. and Segars, A. H. (2001), "Knowledge Management: An Organizational Capabilities Perspective," Journal of Management Information Systems, 18 (1), 185-214.

Granovetter, M. S. (1973), "The Strength of Weak Ties," American Journal Of Sociology (78), 1360-1380.

Hair, J., Anderson, R., Tatharn, R. and Black, W. (1998), Multivariate Data Analysis with Readings, Prentice Hall International, Upper Saddle River, NJ.

Harper, F. M., Raban, D., Rafaeli, S. and Konstan, J. A. (2008), "Predictors of answer quality in online Q\&A sites," in Proc. of CHI.

Hegel, J, W. F. (1977), Phenomenology of spirit (A.V. Miller, Trans.). Oxford, UK: Oxford University Press.

von Hippel, E. (2005), Democratizing Innovation, Cambridge: MIT Press.

Jin, J., Li, Y., Zhong, X. and Zhai, L. (2015), "Why users contribute knowledge to online communities: An empirical study of an online Q\&A community," Information \& Management, (52), 840-849.

Kaptelinin, V. and Nardi, B. A. (2006), Acting with Technology: Activity Theory and Interaction Design, Cambridge, MA: MIT Press.

Knorr Cetina, K. (1997), "Sociality with objects: social relations in post-social knowledge societies," Theory, Culture and Society, 14 (4), 1-30.

Kojève, A. (1969). Introduction to the reading of Hegel. Lectures on the phenomenology of spirit. London: Cornell University Press.

Koufteros, X. A. (1999), "Testing a model of pull production: A paradigm for manufacturing re-search using structural equation modeling," Journal of Operation Management, (17), 467-488.

Lanham, R. A. (2006), The Economics of Attention: Style and Substance in the Age of Information, University of Chicago Press.

Leont'ev, A. N. 1978. Activity, Consciousness, and Personality, Englewood Cliffs, NJ: Prentice Hall.

Lyytinen, K., Yoo, Y., Richard, J. and Boland, J. (2015), 'Digital product innovation within fourclasses of innovation networks', Information Systems Journal, 1-28.

McEvily, B. and Zaheer, A. (1999), "Bridging ties: a Source of firm heterogeneity in competitive capabilities," Strategic Management Journal, 20 (12), 1133-1156.

Miettinen, R. (2005), “Object of activity and individual motivation,” Mind, Culture, Activity 12 (1), 53-69.

Miettinen, R. and Virkkunen, J. (2005), 'Epistemic objects, artefacts, and organizational change', Organization, 12 (3), 437-456.

Nahapiet, J. and Ghoshal, S. (1998), "Social Capital, Intellectual Capital and the Organizational Advantage," Academy of Management Review, 23 (2), 242-266.

Nätti, S. and Halinen, A. and Hanttu, N. (2006), "Customer Knowledge Transfer and Key Account Management in Professional Service Organisations," International Journal of Service Industry Management, 17 (4), 304-319.

Nicolini, D., Mengis, J. and Swan, J. (2012), "Understanding the Role of Objects in Cross-Disciplinary Collaboration," Organization Science 23 (3), 612-629.

Orlikowski, W. J. (2007), "Socio-material practices: Exploring technology at work," Organization Studies, 28 (9), 1435-1448.

Patil, S. and Lee, K. (2016), "Detecting experts on Quora: by their activity, quality of answers, linguistic characteristics and temporal behaviors," Social Network Analysis and Mining 6 (5), 1-11. 
Paul, S. A., Hong, L. and Chi, E. H. (2012), "Who is authoritative? understanding reputation mechanisms in quora," in Proc. of Collective Intelligence (Cambridge, MA, April 2012).

Rennstam, J. (2012), “Object-Control: A Study of Technologically Dense Knowledge Work," Organization Studies, 33 (8), 1071-1090.

Rheinberger, H. (1997), Toward a History of Epistemic Things: Synthesizing Proteins in the Test Tube, Palo Alto, CA: Stanford University Press.

Rheinberger, H. (2005), "A reply to David Bloor: 'Toward a sociology of epistemic things", Perspective in Science, 13 (3), 406-410.

Sigala, M. and Chalkiti, K. (2015), "Knowledge management, social media and employee creativity," International Journal of Hospitality Management (45), 44-58.

Trompette, P., Chanal, V. and Pelissier, C. (2008), "Crowdsourcing as a way to access external knowledge for innovation: Control, incentive and coordination in hybrid forms of innovation," in 24th EGOS Colloquium, Amsterdam, France.

Tsai, W. P. (2001), "Knowledge transfer in inter-organizational networks: Effects of network position and absorptive capacity on innovation," Academy of Management Journal, 44 (5), 996-1004.

Vázquez, X. H. (2006), "An eclectic explanation of shop floor control using efficiency and power theories," Organization Studies, (27): 1421-1446.

Wasko, M. M. and Faraj, S. (2005), "Why should I share? Examining social capital and knowledge contribution in electronic networks of practice," MIS Quarterly, 29 (1), 35-57.

Yang, J. (2005), "Knowledge integration and innovation: Securing new product advantage in high technology industry," Journal of High-Technology Management Research, 16 (1), 121-135.

Yoo, Y., Henfridsson, O. and Lyytinen, K. (2010), "Research Commentary-The New Organizing Logic of Digital Innovation: An Agenda for Information Systems Research," Information Systems Research, 21(4), 724-735.

Yoo, Y., Lyytinen, K. and Boland, R. J. (2008), "Distributed Innovation in Classes of Networks", in Proceedings of the 41st Hawaii International Conference on System Sciences, IEEE. 